\title{
Characterization of antimicrobial peptide genes from Japanese honeybee Apis cerana japonica (Hymenoptera: Apidae)
}

\author{
Mikio YoshiYama* and Kiyoshi KIMURA \\ Honeybee Research Group, Animal Breeding and Reproduction Research Team, National Institute of Livestock and Grassland \\ Science; Tsukuba, Ibaraki 305-0901, Japan
}

(Received 16 April 2010; Accepted 8 July 2010)

\begin{abstract}
Two antimicrobial peptide cDNA clones, abaecin (named AcjAba) and defensin (named AcjDef2) were isolated from the Japanese honeybee, Apis cerana japonica, using rapid amplification of cDNA end (RACE) methods. Deduced amino acid sequences of AcjAba and AcjDef2 in A. c. japonica showed high identity with those in the European honeybee, Apis mellifera (97.1\%, and $93.0 \%$, respectively). In the mature peptide region, only one amino acid residue (Val) of A. mellifera abaecin was replaced with (Ile) of A. c. japonica abaecin. Quantitative real-time RT-PCR was carried out to investigate the expression profiles of $A c j A b a$ and AcjDef 2 after inoculation with Paenibacillus larvae, the causative agent of American foulbrood. The transcription of $A c j A b a$ and $A c j D e f 2$ was not significantly up-regulated in response to exposure to $P$. larvae. As social insects, honeybees have evolved behavioral traits during their evolutionary history to reduce threats from invading pathogens. The relationship between the defensive behaviors and innate immune systems of Japanese honeybees is discussed.
\end{abstract}

Key words: Apis cerana japonica; antimicrobial peptide; American foulbrood; Paenibacillus larvae

\section{INTRODUCTION}

Honeybee colonies are infected by numerous pathogens, including bacteria, fungi, viruses, and parasites; for example, American foulbrood (AFB), caused by Gram-positive bacterium Paenibacillus larvae (Genersch et al., 2006), is one of the most destructive diseases affecting honeybee larvae. AFB is highly infectious and capable of killing honeybee colonies. In addition, the ability to form spores allows $P$. larvae to remain viable for many years and to survive under adverse environmental conditions (Matheson and Reid, 1992). Two antibiotics, oxytetracycline and tylosin, are currently used to control AFB in honeybee colonies, but oxytetracycline-resistant $P$. larvae isolates have been reported around the world (Alippi, 2000; Miyagi et al., 2000; Evans, 2003). AFB leads to a decrease of the honeybee population and honey production, causing serious economic losses and problems for the beekeeping industry (Ellis and Munn, 2005; Genersch, 2010).
With the honeybee population declining in many parts of the world (van Engelsdorp et al., 2008), multiple factors, such as pathogens and other stressors, are likely to be involved in the current colony depopulation. There has been growing interest in the immune defense mechanisms of honeybees concerning the risk of infection by pathogens. To combat invading pathogenic micro-organisms, honeybees possess effective innate immune systems. Antimicrobial peptides are synthesized and secreted in response to bacterial infection. Several antimicrobial peptides, with a broad spectrum of antibiotic activities against a variety of bacteria, have been reported in European honeybee Apis mellifera. Transcript levels of antimicrobial peptides genes, abaecin and defensin, against $P$. larvae infection have been studied (Evans, 2004).

The native honeybee of Japan, Apis cerana japonica, is wildly distributed in Japan. It is characterized by its dark coloring, small size, and gentle behavior. Due to its tolerance to pathogenic micro-organisms and parasitic mites, $A$. c. japonica

\footnotetext{
* To whom correspondence should be addressed at: E-mail: yoshiyam@affrc.go.jp
} DOI: $10.1303 /$ aez.2010.609 
is receiving increased attention with regard to honeybee diseases; however, little $A$. c. japonica genomic-based research has been reported. To gain molecular insight into AFB tolerance, antimicrobial peptide cDNA clones, abaecin and defensin, were isolated from A. c. japonica.

\section{MATERIALS AND METHODS}

Two A. c. japonica colonies were collected independently in the Tsukuba area in May 2009 and September 2009. Another colony was purchased from Matsumaru Honeybee Farm (Ichikawa, Japan). These three colonies were reared in wooden hives in an apiary at the Honeybee Research Group, National Institute of Livestock and Grassland Science. Escherichia coli JM109 $\left(1 \times 10^{6} / \mathrm{ml}\right)$ was injected into A. c. japonica larvae (4th instar). Total RNA was isolated $24 \mathrm{~h}$ after bacterial injection using ISOGEN (Nippon Gene), according to the manufacturer's instructions. To obtain the full-length cDNA, 5' - and 3'RACE was carried out using a First Choice RLM-RACE kit (Ambion). Abaecin specific primers were designed based on A. mellifera genes (GB18323). Primers used were as follows: AcjAba5: 5'-CCT TGA GGC CAT TTA ATT TTC GGA TT'-3' and, AcjAba3: 5'-CAG CAT TCG CGT ATG TAC CAT TAC CAA A-3'. Defensin2-specific primers were derived from the database of the European honeybee A. mellifera (GB10036): AcjDef2-F1: 5'-CAA CTA CCG CCT TTA CGT CGT-3', AcjDef2-F2: 5'-TAA ACA TCT GAT AGC GCA AGC-3', AcjDef2-R: 5'ACC ACT ACG ACA TCG AAG GGT AAC -3'.

The nucleotide sequence was determined by dye terminator cycle sequencing using an ABI PRIZM 377 DNA sequencer (Applied Biosystems). Sequences were aligned by GENETYX-MAC ver 9.0 (Software Development). Signal peptide sequences were searched for using SignalP 3.0 (http://www. cbs.dtu.dk/services/SignalP/). Antimicrobial peptide genes from other insects were retrieved from the GenBank/EMBL database (http://ncbi.nlm.nih. gov/). Amino acid sequence alignments were performed with CLUSTALX (http://bips.u-strasbg.fr/ fr/Documentation/ClustalX/) and used to propose phylogenetic relationships using neighbor-joining algorithms with NJplot (http://pbil.univ-lyon1.fr/ software/njplot.html). The neighbor-joining tree was boot strapped 1,000 times. The DNA data from this study appear in the GenBank, EMBL, and DDBJ databases under accession numbers AB490712AB490717, and AB540993-AB540997.

To analyze transcription levels following natural infection, first instar larvae were inoculated with a final concentration of 100 spores $/ \mu 1$ from pathogenic isolates of $P$. larvae (RIAS No.P-1) and maintained at $34^{\circ} \mathrm{C}$ in 96 -well plates containing liquid medium (Evans, 2004). Control larvae were given the same food without bacteria spores. Total RNA was isolated from individual larvae from each of three colonies using an RNeasy kit (Qiagen) according to the manufacturer's instructions. First-strand cDNA was synthesized from $1 \mu \mathrm{g}$ total RNA using the First Strand cDNA Synthesis kit (Promega) with oligo dT primers. Gene-specific primers (QRT-aba-F: 5'-CCA TTC AAT CCG AAA ATT AAA TGG-3', QRT-aba-R: 5'-TAC GAT TTA TTT CAA TTC ATA ATT-3', QRTdef2-F: 5'-GAG GCA AAT TGA GGA GGA AAA TAT-3', and QRT-def2-R: 5' -CTA AAC ATC TGA TAG CGC AAG CTG-3') were designed for real-time RT-PCR. PCRs were performed in a final volume of $20 \mu \mathrm{l}$ with the LightCycler ${ }^{\circledR} 480$ System (Roche) using the Fast Start Reaction Mix SYBR green 1 (Roche). Cycling conditions were $95^{\circ} \mathrm{C}$ for $5 \mathrm{~min}$ followed by 40 cycles of $95^{\circ} \mathrm{C}$ for $10 \mathrm{~s}, 60^{\circ} \mathrm{C}$ for $5 \mathrm{~s}$, and $72^{\circ} \mathrm{C}$ for $10 \mathrm{~s}$ with transition rates of $20^{\circ} \mathrm{C} / \mathrm{s}$. Specific primers designed for the ribosomal protein gene ( $r p S 5)$ were as follows: QRTrpS5-F: 5'-TTT CCC ATT ATT TCT ACC ATG CAT-3', and QRT-rpS5-R: 5'-CTT TAC AAG ATT ATA TTG CCG TTA-3'. Serial dilutions of the pGEM $^{\circledR}$-T Easy vector containing the rp5S gene were used for the standard curve. Three independent experiments were performed to calculate standard deviations, and transcript levels normalized against the $r p 5 S$ gene were used as internal controls.

\section{RESULTS AND DISCUSSION}

Six different types of $A$. c. japonica abaecin (AcjAbal 6) cDNA were isolated by RACE methods. The deduced AcjAba peptide was 53 amino acids in length, of which the signal peptide was 19 amino acids. AcjAba amino acid sequences were aligned with A. mellifera abaecin (Fig 1A). Compared with European honeybee abaecin peptides (Casteels et al., 1990), only one amino acid 
A

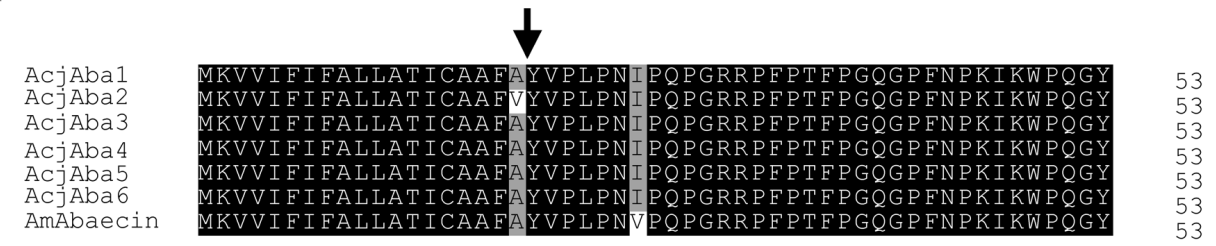

B

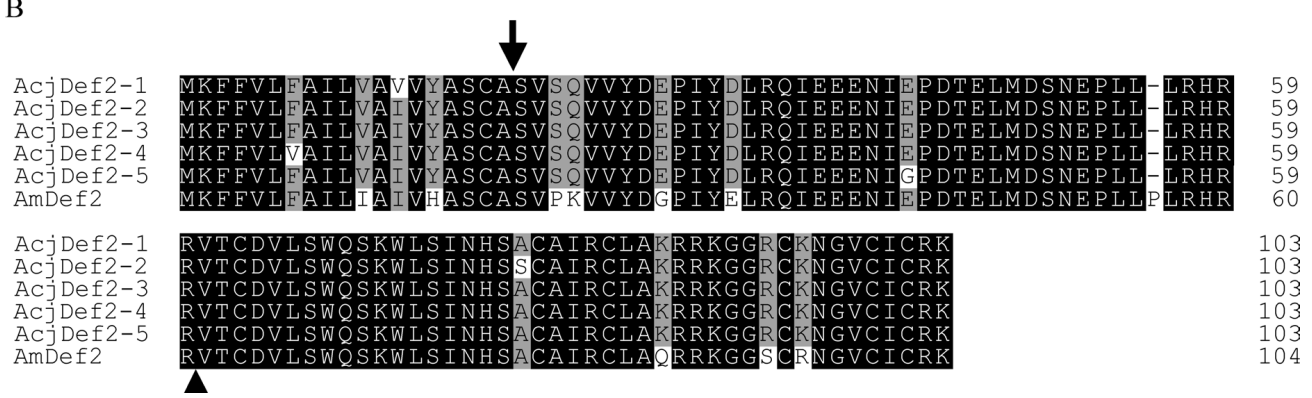

Fig. 1. (A) Comparison of deduced amino acid sequences of A. cerane japonica and A. mellifera abaecins. White uppercase letters on a black background indicate identical amino acid sequences. Different amino acid residues are shown as black uppercase letters on a white background. Arrow indicates the putative cleavage site. Hyphens represent sequence alignment gaps. A. cerane japonica abaecins are abbreviated as AcjAba1 AcjAba6. A. mellifera abaecin is abbreviated as AmAbaecin. (B) Comparison of deduced amino acid sequences of $A$. cerane japonica defensin 2 and $A$. mellifera defensin2. White uppercase letters on a black background indicate identical amino acid sequences. Different amino acid residues are shown as black uppercase letters on a white background. Arrow indicates the putative cleavage site. Arrowhead indicates mature peptide regions. Hyphens represent sequence alignment gaps. A. cerane japonica defencins are abbreviated as AcjDef2-1 AcjDef2-5. A. mellifera defensin is abbreviated as AmDef2.

replacement existed in the mature peptide region of A. c. japonica abaecin. The apolar amino acid Val (V) (TGT) substitutes the apolar amino acid I1e (I) (TAT) at position 26, which may not change its secondary structure and properties. Apidaecin and abaecin belong to the Pro-rich family of antimicrobial peptides (Otvos, 2002). A. c. japonica abaecin consists of 34 peptide residues and 10 prolines. This type of antimicrobial peptide is predominantly active against Gram-negative bacteria by unique modes of action. For example, apidaecin kills bacteria by the initial, non-specific encounter of the peptide with an outer membrane, followed by invasion into the periplasmic space and finally by translocation of the peptide into the interior of the bacteria cell (Li et al., 2006).

Overall, five different defensin 2 cDNA sequences (AcjDef 2-1 5) were cloned and characterized. The deduced AcjDef2 peptide was 103 amino acids long. This gene consisted of a 19 amino acid signal peptide, 41 amino acid propeptide, and a 43 amino acid mature region. These cDNA sequences were highly similar to the defensin2 gene of the Euro- pean honeybee, A. mellifera (Klaudiny et al., 2005) (Fig 1B). Defensins are cationic peptides with molecular masses ranging from 3-5 kDa, containing 6 conserved cysteine residues that form disulfide bonds. Compared with European honeybee defensin 2 peptides, there are 3 nonsynonymous substitutions in the mature peptide region of Japanese honeybee defensin2, Gln (Q) to Lys (K), Ser (S) to $\operatorname{Arg}(\mathrm{R})$, and $\operatorname{Arg}(\mathrm{R})$ to Lys $(\mathrm{K})$ at positions 87, 93, and 95 , respectively. The distribution of positivelycharged amino acid residues is suggested to determine the antimicrobial activity of cationic antimicrobial peptides. It is reported that the replacement of positively-charged residues in defensins reduces bacterial killing significantly (Zou et al., 2007); therefore, the changes to positively charged Arg (R) and Lys (K) may affect the antimicrobial activity of Japanese honeybee defensin2.

In $A$. mellifera, abaecin has shown previously to be up-regulated in response to exposure to $P$. larvae, whereas the defensin 2 gene was not induced (Evans, 2004). In this study, however, both abaecin and defensin 2 of $A$. c. japonica showed no signifi- 
A
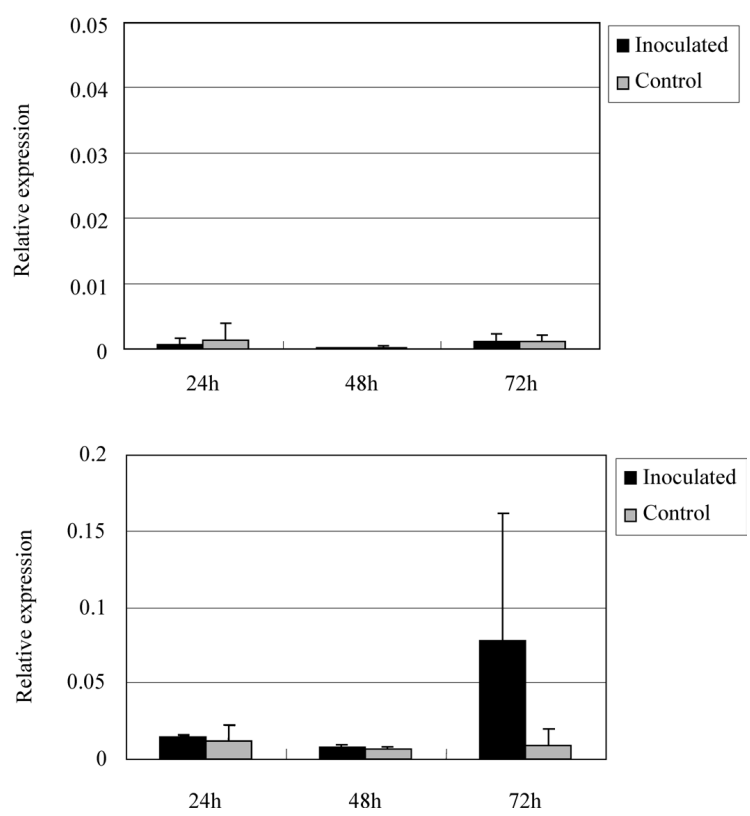

Fig. 2. Transcriptional levels for control larvae and larvae inoculated with $P$. larvae for abaecin (A) and defensin2 (B). Larvae inoculated with $P$. larvae were incubated for 24,48 , and $72 \mathrm{~h}$. An rp5S gene was used as an internal control. Data are the means \pm SEM for three independent experiments.

cant up-regulation after oral inoculation with P. larvae (Fig 2A, B). One reason for the different expression profiles could be genetic variability across individual honeybees in their immune response to bacterial infection (Evans, 2004). A. mellifera abaecin loses most of its antibacterial activity in the presence of phosphate-buffered saline, which resembles the honeybee's physiological environment (Casteels et al., 1990). This weak antimicrobial activity of abaecin seems to function as a back-up for apidaecin-resistant bacteria (Casteels et al., 1993). Recently, it was reported that the response of honeybees to $P$. larvae is involved with another immune effector molecule, hymenoptaecin (Chan et al., 2009). The authors were only able to quantify hymenoptaecin in the hemolymph of $P$. larvae-infected European honeybees. Hymenoptaecin up-regulation after oral inoculation with $P$. larvae was also studied (Evans et al., 2006). Furthermore, Chan et al. (2009) detected increased levels of prophenoloxidase (proPO) and lysozyme in the larval hemolymph, suggesting that ProPO and lysozyme play important roles, involving killing pathogens directly. Neither abaecin nor defensin was detected in honeybee hemolymph in re-
A

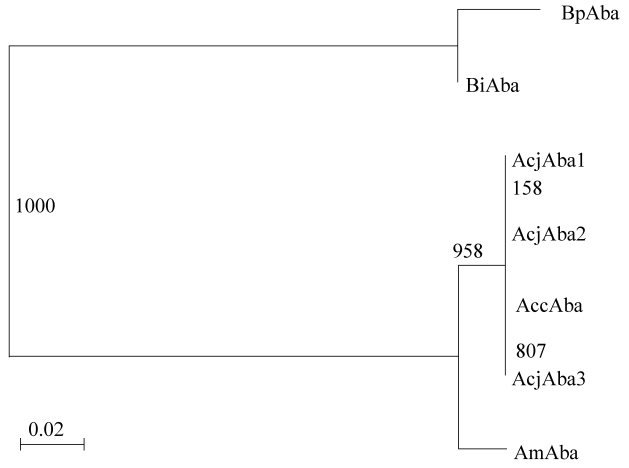

B

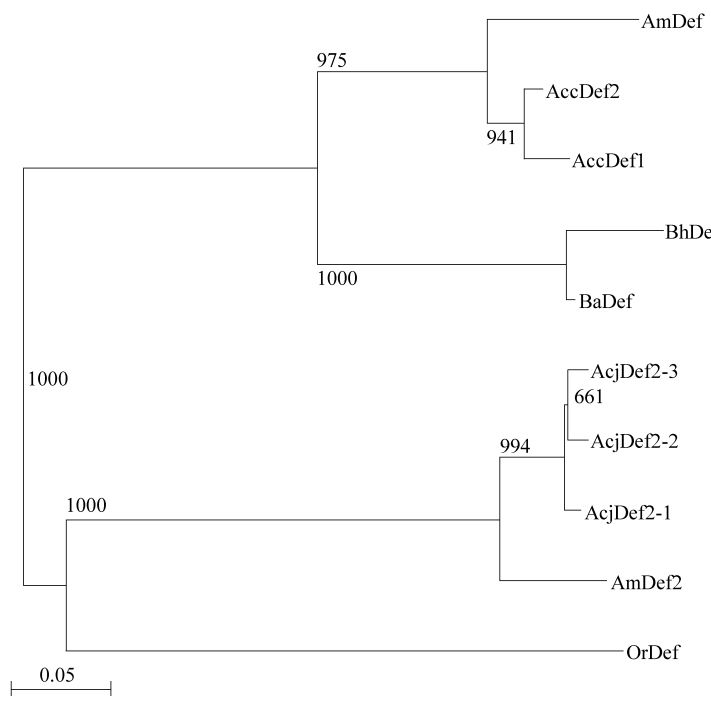

Fig. 3 (A) Phylogenetic analysis of A. c. japonica and related insect abaecins. Amino acid sequences were retrieved from the following databases: BpAba: Bombus pascuorum (P81463), BiAba: Bombus ignitus (AAQ90411), AccAba: Apis cerana cerana (EU714044), and AmAba: A. mellifera (NM_001011617). A phylogenetic tree was constructed using CLUSTAL X. (B) Phylogenetic analysis of AcjDef2 and related insect defensins. The amino acid sequences of mature peptides were retrieved from the following databases: BaDef: Bombus ardens ardens (FJ172505), BhDef: Bombus hypocrita sapporoensis (FJ172504), AccDef1: Apis cerana cerana (EU727268), AccDef2: Apis cerana cerana (EU727269), OrDef: Oryctes rhinoceros defensin (AB011245), AmDef: Apis mellifera (U15955), and AmDef2: A. mellifera (NM_001011638).

sponse to P. larvae infection (Chan et al., 2009), which is in agreement with the results of our gene expression analysis.

Phylogenetic analysis showed that abaecin from $A$. cerana groups, including AcjAba, is closely related to A. mellifera abaecin (Fig 3A). A. cerana 
groups and $A c j D e f 2$ s defensin 2 were defined with high bootstrap values, suggesting that AcjDef 2 is more closely related to coleopteran defensin than to A. cerana defensin2. This implied that honeybee defensins represent two distinct groups (Fig 3B). This differentiation could be influenced by the kinds of pathogens that each species has encountered during their evolutionary history.

Genomic analysis revealed that honeybees, $A$. mellifera, carry fewer known immune genes than Drosophila melanogaster or Anopheles gambiae, suggesting that a compensatory system to secure diseases tolerance exists (Honeybee Genome Sequencing Consortium, 2006). Honeybees have developed several kinds of behavior in order to avoid and eliminate invading pathogens (Cremer et al., 2007). In particular, Eastern honeybees are known to have established defense behaviors, such as heat balling, and social waves against hornet attack (Ono et al., 1995; Kastberger et al., 2008). In addition, A. cerana spp. have developed specific defense behaviors that protect the colony by preventing infectious diseases and parasites, such as AFB and Varroa mites. P. larvae-inoculated larvae were removed by adult worker bees before the capped stage (Chen et al., 2000). A. cerana worker bees are able to remove Varroa mites from their bodies and nest mates by grooming. A. cerana are able to recognize capped brood cells infected by Varroa mites, and can open cells and kill mites by biting (Peng, 1987). Thus, hygienic behaviors could contribute to decreasing the potential risks of spreading viral diseases transmitted by Varroa mites. To speculate the relationship between the innate immune system and social defense behavior in Japanese honeybees, further research is needed to determine the function of other immune effector molecules against $P$. larvae.

\section{ACKNOWLEDGMENTS}

The authors are grateful to Ms. Meihua Wu for her technical assistance. We also thank Dr. Atsuko Kataoka for providing us with a strain of P. l. larvae (RIAS No.P-1).

\section{REFERENCES}

Alippi, A. M. (2000) Is Terramycin ${ }^{\circledR}$ losing its effectiveness against AFB? The Argentinean experience. Bee Biz 11: 27-29.

Casteels, P., C. Ampe, L. Riviere, J. Van Damme, C. Elicone, M. Fleming, F. Jacobs and P. Tempst (1990) Isolation and characterization of abaecin, a major antibacterial response peptide in the honeybee (Apis mellifera). Eur. J. Biochem. 187: 381-386.

Casteels, P., C. Ampe, F. Jacobs and P. Tempst (1993) Functional and chemical characterization of Hymenoptaecin, an antibacterial polypeptide that is infection-inducible in the honeybee (Apis mellifera). J. Biol. Chem. 268: 7044-7054.

Chan, Q. W., A. P. Melathopoulos, S. F. Pernal and L. J. Foster (2009) The innate immune and systemic response in honey bees to a bacterial pathogen, Paenibacillus larvae. BMC Genomics 10: 387.

Chen, Y. W., C. H. Wang, J. An and K. K. Ho (2000) Susceptibility of the Asian honey bee, Apis cerana, to American foulbrood, Paenibacillus larvae larvae. J. Apic. Res. 39: 169-175.

Cremer, S., S. A. Armitage and P. Schmid-Hempel (2007) Social immunity. Curr. Biol. 17: R693-702.

Ellis, J. D. and P. A. Munn (2005) The worldwide health status of honey bees. Bee World 86: 88-101.

Evans, J. D. (2003) Diverse origins of tetracycline resistance in the honey bee bacterial pathogen Paenibacillus larvae. J. Invertebr. Pathol. 83: 46-50.

Evans, J. D. (2004) Transcriptional immune responses by honey bee larvae during invasion by the bacterial pathogen, Paenibacillus larvae. J. Invertebr. Pathol. 85: $105-111$

Evans, J. D., K. Aronstein, Y. P. Chen, C. Hetru, J. L. Imler, H. Jiang, M. Kanost, G. J. Thompson, Z. Zou and D. Hultmark (2006) Immune pathways and defence mechanisms in honey bees Apis mellifera. Insect Mol. Biol. 15: 645-656.

Genersch, E. (2010) American Foulbrood in honeybees and its causative agent, Paenibacillus larvae. J. Invertebr. Pathol. 103: S10-S19.

Genersch, E., E. Forsgren, J. Pentikäinen, A. Ashiralieva, S. Rauch, J. Kilwinski and I. Fries (2006) Reclassification of Paenibacillus larvae subsp. Pulvifaciens and Paenibacillus larvrae subsp. Larvae as Paenibacillus larvae without subspecies differentiation. Int. J. Syst. Evol. Microbiol. 56: 501-511.

Honeybee Genome Sequencing Consortium (2006) Insights into social insects from the genome of the honeybee Apis mellifera. Nature 443: 931-949.

Kastberger, G., E. Schmelzer and I. Kranner (2008) Social waves in giant honeybees repel hornest. PLoS One 3: e3141.

Klaudiny, J., S. Albert, K. Bachanova, J. Kopernicky and J. Simuth (2005) Two structurally different defensin genes, one of them encoding a novel defensin isoform, are expressed in honeybee Apis mellifera. Insect Biochem. Mol. Biol. 35: 11-22.

Li, W. F., G. X. Ma and X. X. Zhou (2006) Apidaecin-type peptides: biodiversity, structure-function relationships and mode of action. Peptides 27: 2350-2359.

Matheson, A. and M. Reid (1992) Strategies for the prevention and control of American foulbrood. Parts I, II, and III. Am. Bee J. 132: 399-402; 133: 471-475; 143: 534 547.

Miyagi, T., C. Y. S. Peng, R. Y. Chuang, E. C. Mussen, M. S. 
Spivak and R. H. Doi (2000) Verification of oxytetracycline-resistant American foulbrood pathogen Paenibacillus larvae in the United States. J. Invertebr. Pathol. 75: 95-96.

Ono, M., T. Igarashi, E. Ohno and M. Sasaki (1995) Unusual thermal defence by a honeybee against mass attack by hornets. Nature 377: 334-336.

Otvos, L. Jr. (2002) The short proline-rich antibacterial peptide family. Cell Mol. Life Sci. 59: 1138-1150.

Peng, C. Y. S., Y. Fang, S. Xu and L. Ge (1987) The resist- ance mechanism of the Asian honey bee, Apis cerana Fabr., to an ectoparasitic mite, Varroa jacobsoni Oudemans. J. Invertebr. Pathol. 49: 54-60.

van Engelsdorp, D., J. Hayes Jr., R. M. Underwood and J. Pettis (2008) A survey of honey bee colony losses in the U.S., Fall 2007 to Spring 2008. PLoS One 3: e4071.

Zou, G., E. de Leeuw, C. Li, M. Pazgier, C. Li, P. Zeng, W. Y. Lu, J. Lubkowski and W. Lu (2007) Toward understanding the cationicity of defensins. Arg and Lys versus their noncoded analogs. J. Biol. Chem. 282: 19653-19665. 